\title{
Independent and opposite associations of serum levels of omentin-1 and adiponectin with increases of glycaemia and incident type 2 diabetes in an older population: KORA F4/FF4 study
}

\author{
Christian Herder ${ }^{1,2}$, Julia M Kannenberg ${ }^{1,2}$, Corinna Niersmannn ${ }^{1,2}$, Cornelia Huth ${ }^{2,3}$, \\ Maren Carstensen-Kirberg ${ }^{1,2}$, Clemens Wittenbecher ${ }^{2,4}$, Matthias Schulze ${ }^{2,4}$, \\ Matthias Blüher ${ }^{2,5,6}$, Wolfgang Rathmann ${ }^{2,7}$, Annette Peters ${ }^{2,3}$, Michael Roden ${ }^{1,2,8}$, \\ Christa Meisinger ${ }^{3, *}$ and Barbara Thorand ${ }^{2,3, *}$
}

${ }^{1}$ Institute for Clinical Diabetology, German Diabetes Center, Leibniz Center for Diabetes Research at Heinrich Heine University Düsseldorf, Düsseldorf, Germany, ${ }^{2}$ German Center for Diabetes Research (DZD), München-Neuherberg, Germany, ${ }^{3}$ Institute of Epidemiology II, Helmholtz Zentrum München, German Research Center for Environmental Health, Neuherberg, Germany, ${ }^{4}$ Department of Molecular Epidemiology, German Institute of Human Nutrition Potsdam-Rehbruecke, Nuthetal, Germany, ${ }^{5}$ Department of Medicine, University of Leipzig, Leipzig, Germany, ${ }^{6} \mathrm{IFB}$ Adiposity Diseases, University of Leipzig, Leipzig, Germany, ${ }^{7}$ Institute for Biometrics and Epidemiology, German Diabetes Center, Leibniz Center for Diabetes Research at Heinrich Heine University Düsseldorf, Düsseldorf, Germany, and ${ }^{8}$ Division of Endocrinology and Diabetology, Medical Faculty, Heinrich Heine University Düsseldorf, Düsseldorf, Germany

*(C Meisinger and B Thorand contributed equally to this work)

Correspondence should be addressed to $\mathrm{C}$ Herder Email

christian.herder@ddz. uni-duesseldorf.de

\begin{abstract}
Objective: Cross-sectional studies found that higher levels of the novel adipokine omentin-1 were associated with higher adiponectin and lower levels of risk factors for type 2 diabetes, but its relevance for incident type 2 diabetes is currently not understood. Therefore this study investigated whether serum omentin-1 was associated with changes in glycaemia and incident type 2 diabetes independently of adiponectin.

Design and methods: The study was based on participants aged 62-81 years from the population-based Cooperative Health Research in the Region of Augsburg (KORA) F4/FF4 cohort. Associations of baseline serum levels of omentin-1 and adiponectin with changes in glycaemia were assessed in 471 non-diabetic participants, and associations between both adipokines and incident type 2 diabetes were assessed in 76 cases and 430 non-cases (follow-up time 6.5 years). Multivariable linear and logistic regression models were adjusted for multiple potential confounders. Results: Higher serum levels of omentin-1 were associated with increases in fasting glucose, 2-h glucose and HbA1c (all $P<0.001)$ and with incident type 2 diabetes (adjusted odds ratio $(\mathrm{OR})(95 \% \mathrm{Cl}): 1.40(1.03 ; 1.90)$ per S.D. of $\log _{2}$-transformed omentin-1; $P=0.032$ ). These associations were independent from adiponectin levels, which showed associations with changes in glycaemia and risk of type 2 diabetes in the opposite direction. We found no statistically significant interactions of omentin-1 with adiponectin or sex in the association with incident type 2 diabetes
\end{abstract} (all $P>0.1$ ).

Conclusions: Systemic levels of omentin-1 were positively associated with increases in glycaemia and incident type 2 diabetes in this older population. These associations were independent of potential confounders including adiponectin.

(C) 2017 European Society of Endocrinology Printed in Great Britain
European Journal of Endocrinology

(2017) 177, 277-286 


\section{Introduction}

Omentin-1 (also known as intelectin-1) was initially discovered as an adipokine with insulin-sensitising properties, which is predominantly expressed in the stromal vascular fraction of visceral adipose tissue (1). Cross-sectional studies found that circulating levels of omentin-1 are positively correlated with those of adiponectin $(2,3,4,5)$. Further similarities between both adipokines extend to their associations with anthropometric and metabolic traits. They are both downregulated in obese individuals and have been found to be lower in people with insulin resistance and type 2 diabetes $(3,6,7,8,9,10,11,12)$.

A cross-sectional analysis in the Cooperative Health Research in the Region of Augsburg (KORA) F4 study confirmed known associations between omentin-1, obesity and insulin resistance. However omentin-1 levels did not differ between individuals with normal glucose tolerance, prediabetes and type 2 diabetes in an analysis adjusted for multiple confounders. Moreover, the aforementioned associations with obesity and diabetesrelated traits were attenuated when associations were adjusted for adiponectin (4).

The relevance of systemic omentin-1 levels for the incidence of type 2 diabetes has only been assessed prospectively in the European Prospective Investigation into Cancer and Nutrition (EPIC)-Potsdam cohort so far. Despite favourable cross-sectional associations between omentin-1 levels and risk factors of diabetes, the prospective part of the study revealed that higher baseline omentin-1 levels were associated with higher rather than lower risk of type 2 diabetes (5). This association was modified by adiponectin levels (5), which are inversely associated with risk of type 2 diabetes in most populationbased studies (13).

Thus, it is currently not well understood to what extent omentin-1 and adiponectin differ in their associations with incident type 2 diabetes, whether these associations are independent from each other or whether both adipokines interact with respect to diabetes risk. It is further unknown if any potential relationship between omentin- 1 and diabetes risk is driven by associations between omentin-1 and fasting or postprandial hyperglycaemia, which would have implications for the underlying pathophysiological mechanism.

The aims of this study were therefore to use data from a well-phenotyped cohort (i) to compare associations of baseline omentin-1 and adiponectin with changes in quantitative parameters of glycaemia (fasting glucose, 2-h glucose from oral glucose tolerance tests (OGTT), HbA1c) in non-diabetic individuals and (ii) to assess the associations of baseline omentin-1 and adiponectin with incident type 2 diabetes after extensive adjustment for confounders.

\section{Subjects and methods}

\section{Study population}

The data are based on the KORA F4 study (2006-2008) and the KORA FF4 study (2013-2014); both are follow-up examinations of the population-based KORA S4 study (1999-2001) conducted in Augsburg (Germany) and two surrounding counties (14). All investigations were performed in accordance with the Declaration of Helsinki, including obtaining written informed consent from all participants. The study was approved by the ethics committee of the Bavarian Chamber of Physicians (Munich, Germany).

The KORA F4 study and the standardised assessment of anthropometric and metabolic variables and lifestyle factors have been described in detail $(4,15)$. Briefly, body mass index (BMI) was calculated using height and weight measured at the study centre. Smoking habits, alcohol consumption and physical activity were assessed in standardised interviews. The calculation of the estimated glomerular filtration rate (eGFR) was performed using the chronic kidney disease epidemiology (CKD-EPI) creatinine equation (16).

Participants without previously diagnosed diabetes (defined as self-report that could be validated by the responsible physician, or as current use of glucoselowering agents) received a standard 75-g OGTT in both KORA F4 and KORA FF4. Incident type 2 diabetes in KORA FF4 was defined based on (i) a self-reported diagnosis of type 2 diabetes since KORA F4 that could be validated by questioning the participants' responsible physicians or medical chart review, (ii) current use of glucose-lowering drugs, or (iii) fasting and/or 2-h glucose levels in the OGTT in the diabetic range (17).

This study included all participants aged $62-81$ years in KORA F4 $(n=1161)$, representing $70.2 \%$ of the study participants from the age range $55-74$ years $(n=1653)$ in the population-based KORA S4 survey (14). The exclusion of individuals with unclear glucose tolerance status, type 1 diabetes or other missing covariables in KORA F4 and of individuals for whom follow-up data from KORA FF4 
were not available resulted in a sample size of 638 individuals (Figs 1 and 2). The analysis of incident type 2 diabetes as outcome was based on 506 individuals (Fig. 1). The mean follow-up time ( \pm s.D.) for this sample was $6.46 \pm 0.22$ years. The analysis of changes in parameters of glycaemia was based on 471 individuals (Fig. 2). The mean follow-up time $( \pm$ s.D.) for this sample was $6.45 \pm 0.22$ years.

\section{Laboratory measurements}

Glucose levels were assessed using the hexokinase method on a Dimension RxL instrument (Dade Behring, Newark, NJ, USA) in KORA F4 and by enzymatic, colorimetric methods on a Dimension Vista 1500 instrument (Siemens Healthcare Diagnostics) or on a Cobas c702 instrument (Roche Diagnostics). The measurement instrument changed from Siemens to Roche halfway during the KORA FF4 study. Calibration formulas were developed using

\section{KORA F4 (2006-2008)}

$n=1161$ aged $62-81$ years

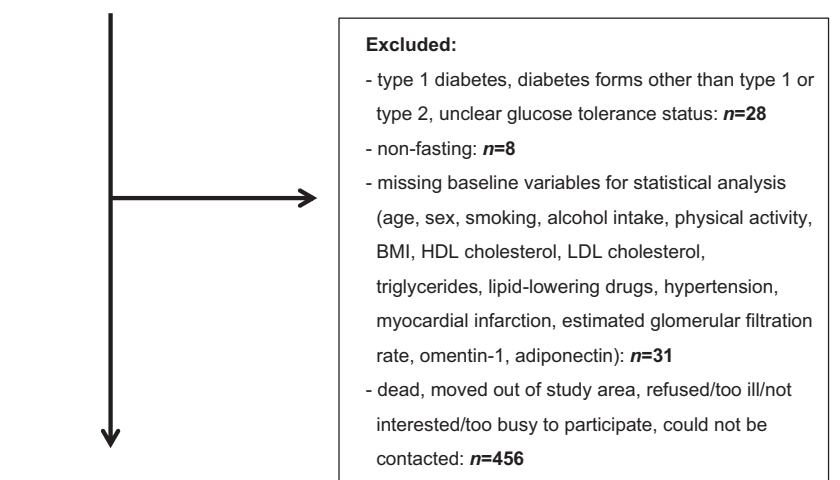

KORA FF4 (2013/2014)

$n=638$

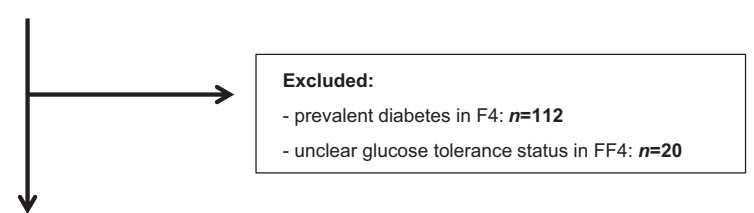

Analysis sample

$n=506$

$n=430$ without incident type 2 diabetes in FF4

$n=76$ with incident type 2 diabetes in FF4

\section{Figure 1}

Description of the study design and population for the analysis of incidence of type 2 diabetes.
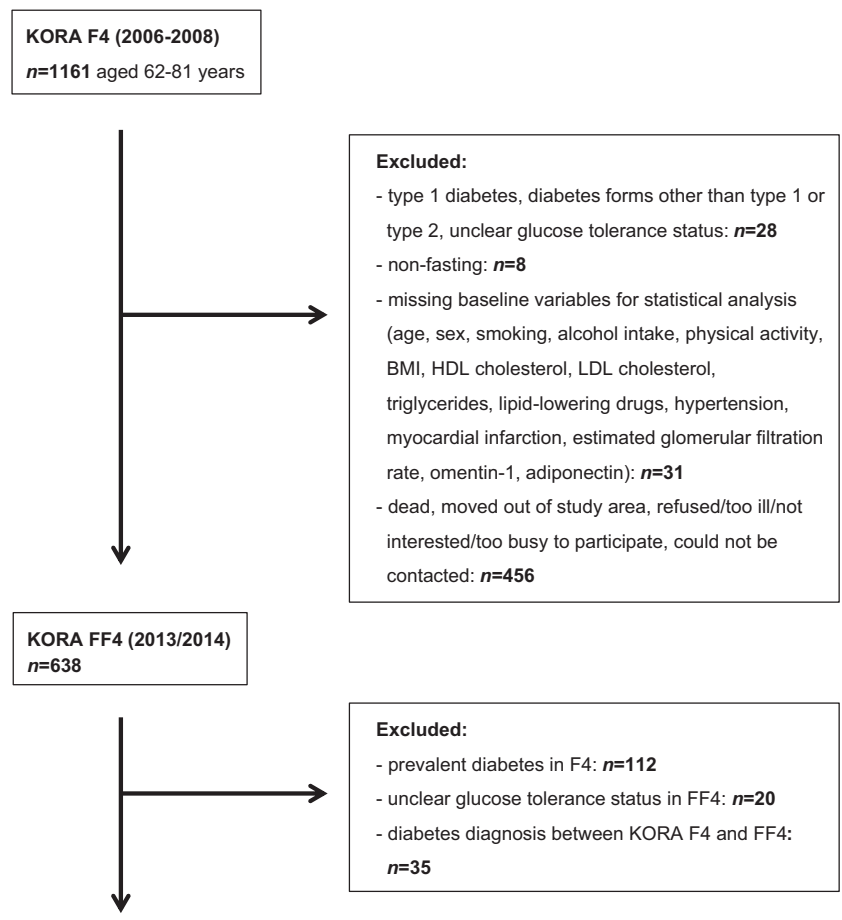

Analysis sample

$n=471$

Figure 2

Description of the study design and population for the analysis of changes in glycaemia between KORA F4 and KORA FF4.

122 KORA FF4 samples, which were measured with both methods during the time of the change. No calibration was needed for glucose because the double measurements showed that the intercept and the slope of the PassingBablok regression used for calibration were estimated to be zero and one, respectively.

HbA1c was measured using cation-exchange highperformance liquid chromatographic, photometric assays on an Adams HA-8160 haemoglobin analysis system (Menarini Diagnostics, Florence, Italy) in KORA F4 and on a VARIANT II TURBO Hemoglobin Testing System (Bio-Rad Laboratories) in KORA FF4 (18).

Serum lipids were measured in KORA F4 using a Dimension RxL instrument (Dade Behring) as described previously (18).

Serum levels of omentin-1 and adiponectin were measured using the Human Omentin-1 ELISA (BioVendor, Brno, Czech Republic) and the Human Total Adiponectin/ Acrp30 Quantikine ELISA Kit (R\&D Systems) with intraassay CVs of $2.0 \%$ and $4.0 \%$ respectively, and inter-assay CVs of 3.8 and $8.0 \%$ respectively $(4,19)$. 


\section{Statistical analysis}

Baseline characteristics of the study samples are given as mean \pm standard deviation (s.D.), median (25th percentile; 75th percentile) or percentages. Groups stratified by diabetes incidence were compared with logistic regression analysis (likelihood ratio tests comparing models with the respective variable, age and sex as independent variables to models with age and sex only).

Associations of omentin-1 and adiponectin with absolute changes in parameters of glycaemia were estimated by multivariable linear regression for the follow-up values of glycaemia parameters from KORA FF4 as dependent variables and both serum omentin- 1 and adiponectin as independent variables in a joint model (adjusting for the baseline level of the respective glycaemia parameter). The analysis used a predefined set of potential confounders based on previous KORA analyses (4) as indicated in the table footnotes. Odds ratios (ORs) and the corresponding 95\% CIs were estimated based on omentin-1 and adiponectin concentrations that were first $\log _{2}$-transformed and then z-standardised so that the unit of exposure corresponds to one S.D. of the $\log _{2}$-transformed levels of the respective biomarker. The continuous outcomes (fasting and 2-h glucose, HbA1c) were only z-standardised.

The association between serum omentin-1 and adiponectin as continuous variables and incident type 2 diabetes was assessed by multivariable logistic regression in a mutually adjusted model using serum omentin- 1 and adiponectin (both $\log _{2}$-transformed and z-standardised), and the aforementioned covariables as independent variables. Potential interactions between omentin-1 and adiponectin were assessed using the interaction term omentin-1*adiponectin in the logistic regression models. Interactions of omentin-1 or adiponectin with sex were assessed using the interaction term omentin$1{ }^{*}$ sex or adiponectin*sex in the logistic regression models. Additionally, analyses were performed stratified by sex or by high vs low levels of omentin- 1 and adiponectin using sexspecific median values of both adipokines for stratification.

All statistical analyses were performed with $R$ version 3.2.4 (R Core Team, R Foundation for Statistical Computing, Vienna, Austria). A $P$ value $<0.05$ was considered to indicate statistical significance.

\section{Results}

\section{Study population and drop-out analyses}

This study is based on participants aged 62-81 years from the KORA F4 study, the follow-up examination of the population-based KORA S4 study (14). Baseline (S4) characteristics of participants and non-participants in F4 have been reported before (15) with nonparticipants characterised by older age and less favourable cardiometabolic risk factors.

We performed a drop-out analysis with KORA F4 as baseline which yielded very similar results as the previously published drop-out analysis for an almost identical population within KORA F4 (20). Briefly, nonparticipants in FF4 were also older and had higher levels of several cardiometabolic risk factors than participants (20). However participants $(n=638)$ did not differ from non-participants $(n=456)$ with respect to their omentin-1 levels (median (25th; 75th percentiles) 479 (403; 501) $\mathrm{ng} / \mathrm{mL}$ vs $497(403 ; 592) \mathrm{ng} / \mathrm{mL}$ respectively; $P=0.217)$ or their adiponectin levels $(10.00(6.38 ; 15.21) \mu \mathrm{g} / \mathrm{mL}$ vs $10.30(6.79 ; 15.07) \mu \mathrm{g} / \mathrm{mL}$ respectively; $P=0.114)$.

\section{Omentin-1, adiponectin and changes in glycaemia}

The analysis of associations between baseline levels of omentin-1 and adiponectin with changes in glycaemia during the 6.5-year follow-up was based on 471 study participants, who neither had type 2 diabetes at baseline nor a diagnosis of type 2 diabetes between the baseline and follow-up examinations (Fig. 2). Table 1 provides a description of their baseline characteristics. Associations between omentin-1 and anthropometric and metabolic variables in KORA F4 have been described for a larger sample (4). Correlations of omentin- 1 and adiponectin on the one hand and demographic, anthropometric, metabolic and lifestyle factors on the other hand in the current study sample $(n=471)$ are provided in Supplementary Tables 1 and 2 (see section on supplementary data given at the end of this article). Both serum omentin-1 and adiponectin were higher in women than in men (both $P<0.01$ ), and serum levels of both adipokines showed a moderately strong correlation $(r=0.32, P<0.01)$.

During the follow-up period, mean fasting glucose levels increased from $5.35 \pm 050$ to $5.66 \pm 0.82 \mathrm{mmol} / \mathrm{L}$ $(P<0.001)$, mean 2-h glucose levels increased from $6.52 \pm 1.62$ to $7.33 \pm 2.43 \mathrm{mmol} / \mathrm{L}(P<0.001)$ and mean HbA1c levels increased from $5.53 \pm 0.29$ to $5.59 \pm 0.43 \%$ $(P<0.001)$.

Higher baseline levels of omentin-1 were associated with stronger increases in all three measures of glycaemia (all $P_{\text {model } 1}<0.001$ ) (Table 2). A 1-s.D. increase in baseline $\log _{2}$-transformed levels of omentin- 1 was associated with increased changes in fasting glucose, 2-h glucose and $\mathrm{HbA1c}$ of 0.173 (95\% CI: 0.098; 0.248) S.D., 0.156 
Table 1 Baseline characteristics of the KORA F4 study population for the analysis of changes in glycaemia between KORA F4 and KORA FF4. Data are given as mean \pm s.D., median and 25th; 75th percentiles or percentages.

\begin{tabular}{l}
\hline Variable \\
\hline$n$ \\
Age (years) \\
Sex $(\%$ male) \\
BMI (kg/m²) \\
Fasting glucose (mmol/L) \\
2-h glucose (mmol/L) \\
HbA1c (\%) \\
HbA1c (mmol/mol) \\
Glucose tolerance status: NGT/IFG/IGT/ \\
IFG \&IGT (\%) \\
Hypertension (\%) \\
Total cholesterol (mmol/L) \\
LDL cholesterol (mmol/L) \\
HDL cholesterol (mmol/L) \\
Triglycerides (mmol/L) \\
Use of lipid-lowering drugs (\%) \\
History of myocardial infarction (\%) \\
eGFR (mL/min per $\left.1.73 \mathrm{~m}^{2}\right)^{\mathrm{c}}$ \\
Smoking (never/former/current) (\%) \\
Physically active (\%) ${ }^{\mathrm{d}}$ \\
Alcohol (none/moderate/high) $(\%)^{\mathrm{e}}$ \\
Adiponectin ( $\mu \mathrm{gg} / \mathrm{mL}$ ) \\
Omentin-1 (ng/mL)
\end{tabular}

\begin{tabular}{c}
\multicolumn{1}{c}{ Values } \\
\hline \multicolumn{1}{c}{471} \\
$68.6 \pm 4.9$ \\
51.6 \\
$27.7 \pm 4.0$ \\
$5.35 \pm 0.50$ \\
$6.52 \pm 1.62$ \\
$5.53 \pm 0.29$ \\
$37 \pm 3$
\end{tabular}

$54.6 / 24.0 / 11.0 / 10.4$

52.4
$5.08 \pm 0.86$
$3.01 \pm 0.71$
$1.46 \pm 0.32$
$1.31 \pm 0.61$
20.0
4.5
$79.7 \pm 13.5$
$53.1 / 39.9 / 7.0$
59.4
$28.0 / 62.2 / 9.8$
$10.31(7.21 ; 15.65)$
$478(409 ; 572)$

aHypertension was defined as blood pressure of $140 / 90 \mathrm{mmHg}$ or higher, or antihypertensive medication given that the participants were aware of being hypertensive; ${ }^{b}$ participants using lipid-lowering drugs were excluded ( $n=101)$; ' the estimated glomerular filtration rate (eGFR) was calculated using the chronic kidney disease epidemiology (CKD-EPI) creatinine equation; ${ }^{d}$ participants were classified as physically active if they participated in sports in summer and in winter and if they were active for $>1 \mathrm{~h} /$ week in at least one season; ${ }^{e}$ moderate defined as $>0$ and $<40 \mathrm{~g} /$ day for men and $>0$ and $<20 \mathrm{~g} /$ day for women, and high defined as $\geq 40 \mathrm{~g} /$ day for men and $\geq 20 \mathrm{~g} /$ day for women.

$\mathrm{BMI}$, body mass index; eGFR, estimated glomerular filtration rate; IFG, impaired fasting glucose; IGT, impaired glucose tolerance; NGT, normal glucose tolerance; T2D, type 2 diabetes.

(95\% CI: $0.075 ; 0.238$ ) s.D. and 0.150 (95\% CI: 0.072; $0.253)$ S.D. respectively. Adjustment for age, sex, and anthropometric and metabolic variables slightly strengthened these associations to effect sizes of between $0.173(0.097 ; 0.254)$ and $0.198(0.123 ; 0.273)$ s.D. in model 4 (all $P<0.001$ ) (Table 2).

In contrast, higher baseline levels of adiponectin were associated with decreases in all three measures of glycaemia ( $P_{\text {model 1 }}$ between $<0.001$ and 0.011). Unlike omentin-1, associations for adiponectin were attenuated with more extensive adjustment for confounders with BMI having the most pronounced effect (Table 2). In the fully adjusted model, a 1-s.D. increase in baseline adiponectin remained associated with decreases in 2-h glucose by 0.103 (0.002;
$0.204)$ S.D. $\left(P_{\text {model } 4}=0.046\right)$ and decreases in HbA1c by $0.101(0.004 ; 0.198)$ s.D. $\left(P_{\text {model } 4}=0.041\right)$.

\section{Omentin-1, adiponectin and incident type 2 diabetes}

The associations of omentin-1, adiponectin and incident type 2 diabetes were assessed in 506 initially non-diabetic study participants, of whom 76 developed type 2 diabetes during the follow-up (Fig. 1). As described before for an almost identical study sample (20), participants with incident type 2 diabetes after follow-up had higher baseline levels of BMI and glycaemia than non-cases. Cases were also more likely to have hypertension and less likely to be physically active at baseline. Omentin-1 levels were $509(420 ; 566) \mathrm{ng} / \mathrm{mL}$ in cases and $476(402 ; 566) \mathrm{ng} /$ $\mathrm{mL}$ in non-cases.

Logistic regression analyses showed that higher baseline omentin-1 was associated with an increased risk of type 2 diabetes at all levels of adjustment, whereas higher baseline adiponectin was associated with a decreased risk (Table 3). Adjustment had almost no impact on effect sizes, and the OR and 95\% CI in the fully adjusted model 4 were $1.40(1.03 ; 1.90)$ for omentin-1 and $0.60(0.42 ; 0.85)$ for adiponectin per 1 -s.D. increases in $\log _{2}$-transformed levels of these adipokines.

There was no significant interaction between omentin-1 and adiponectin in their association with diabetes risk ( $P_{\text {interaction }}$ between 0.112 and 0.401 for models 1-4) (Table 3). Supplementary Figure 1 shows the associations between high and low levels of omentin-1 with incident type 2 diabetes stratified by adiponectin levels (high vs low) and vice versa.

In a first sensitivity analysis, conducted for the fully adjusted model 4 , we stratified the study population by sex. These analyses showed similar effect sizes for men, women and the combined study population for both adipokines, although the sex-stratified association results were only significant for adiponectin (Supplementary Table 3). However there was no significant interaction between either adipokine or sex in the association with incident type 2 diabetes (Supplementary Table 3, model 4).

In a second sensitivity analysis, we extended model 4 by adjustment for baseline fasting glucose and 2-h glucose. This further adjustment had almost no impact on effect sizes for omentin-1, which remained significantly associated with incident type 2 diabetes in the total study population (OR (95\% CI): 1.47 (1.01; 2.13), $P=0.043)$, but not in men and women separately (Supplementary Table 3, model 5). In contrast, 
Table 2 Associations of serum levels of omentin-1 and adiponectin (per S.D.) with changes in quantitative parameters of glycaemia.

\begin{tabular}{|c|c|c|}
\hline Variable & Model & Omentin-1 $\beta(95 \% \mathrm{Cl})$ \\
\hline \multirow[t]{4}{*}{ Fasting glucose (mmol/L) } & 1 & $0.173(0.098 ; 0.248)$ \\
\hline & 2 & $0.186(0.109 ; 0.236)$ \\
\hline & 3 & $0.193(0.119 ; 0.267)$ \\
\hline & 4 & $0.198(0.123 ; 0.273)$ \\
\hline \multirow[t]{4}{*}{ 2-h glucose (mmol/L) } & 1 & $0.156(0.075 ; 0.238)$ \\
\hline & 2 & $0.172(0.089 ; 0.255)$ \\
\hline & 3 & $0.177(0.097 ; 0.258)$ \\
\hline & 4 & $0.173(0.097 ; 0.254)$ \\
\hline \multirow[t]{4}{*}{ HbA1c (\%) } & 1 & $0.150(0.072 ; 0.228)$ \\
\hline & 2 & $0.166(0.087 ; 0.245)$ \\
\hline & 3 & $0.171(0.093 ; 0.248)$ \\
\hline & 4 & $0.175(0.097 ; 0.253)$ \\
\hline
\end{tabular}

\begin{tabular}{cc}
\hline $\boldsymbol{P}$ \\
\hline$<0.001$ \\
$<0.001$ \\
$<0.001$ \\
$<0.001$ \\
$<0.001$ \\
$<0.001$ \\
$<0.001$ \\
$<0.001$ \\
$<0.001$ \\
$<0.001$ \\
$<0.001$ \\
$<0.001$ \\
\hline
\end{tabular}

\begin{tabular}{l}
\hline Adiponectin $\beta(95 \% \mathrm{Cl})$ \\
\hline$-0.102(-0.181 ;-0.023)$ \\
$-0.103(-0.192 ;-0.014)$ \\
$-0.049(-0.136 ; 0.039)$ \\
$-0.034(-0.127 ; 0.059)$ \\
$-0.161(-0.244 ;-0.079)$ \\
$-0.168(-0.265 ;-0.072)$ \\
$-0.113(-0.209 ;-0.017)$ \\
$-0.103(-0.204 ;-0.002)$ \\
$-0.139(-0.217 ;-0.062)$ \\
$-0.179(-0.269 ;-0.089)$ \\
$-0.134(-0.225 ;-0.043)$ \\
$-0.101(-0.198 ;-0.004)$ \\
\hline
\end{tabular}

$\begin{array}{r}\multicolumn{1}{c}{\boldsymbol{P}} \\ \hline 0.011 \\ 0.024 \\ 0.276 \\ 0.476 \\ <0.001 \\ 0.001 \\ 0.021 \\ 0.046 \\ <0.001 \\ <0.001 \\ 0.004 \\ 0.041 \\ \hline\end{array}$

The regression coefficients $\beta$ can be interpreted as the change in glycaemia per S.D. of the respective outcome for a 1-S.D. difference in log ${ }_{2}$-transformed omentin-1 or adiponectin level at baseline. Model 1: adjusted for respective baseline value (KORA F4) of glycaemia. Model 2: model 1+age, sex, smoking, alcohol consumption, physical activity. Model 3: model 2+BMI. Model 4: model 3+ HDL cholesterol, LDL cholesterol, triglycerides, use of lipid-lowering drugs, hypertension, prevalent myocardial infarction, estimated glomerular filtration rate.

associations between adiponectin and incident type 2 diabetes were attenuated (total study sample, model 5: OR (95\% CI) $0.74(0.49 ; 1.13), P=0.168)$. Interactions by sex were also not significant in this model (Supplementary Table 3).

\section{Discussion}

This study shows that associations between serum levels of omentin-1 and adiponectin were associated with increases in glycaemia and incident type 2 diabetes in opposite directions. The associations were positive for omentin-1 and inverse for adiponectin, independent from one another and not substantially altered by adjusting for multiple potential confounders.

\section{Associations between omentin-1, adiponectin and increases in glycaemia}

This is the first study to demonstrate that higher systemic omentin-1 levels are associated with future increases in fasting glucose, 2-h glucose and HbA1c. There are several aspects of this first main finding of our study that are noteworthy.

First, the direction of the longitudinal associations between omentin-1 and glycaemia differed markedly from the direction observed in cross-sectional analyses of the KORA F4 study and other populations (4). In the KORA F4 study, omentin-1 was inversely associated with 2-h glucose in models adjusting for age, sex, BMI and lifestyle factors. For omentin-1 and fasting glucose and/ or HbA1c, several small studies using different omentin-1 assays reported inverse associations $(12,21)$, whereas the KORA F4 and EPIC-Potsdam studies using the same assay observed no relationships $(4,5)$.

Second, it is remarkable that the associations between baseline omentin-1 and increases in glycaemia were robust to confounding by age, sex, and anthropometric, metabolic and lifestyle factor variables. In contrast, cross-sectional associations between omentin-1 and 2-h glucose in KORA F4 were considerably attenuated by adjustment especially for BMI and serum lipids (4).

Table 3 Associations of serum levels of omentin-1 and adiponectin (per s.D.) with incident type 2 diabetes.

\begin{tabular}{l} 
Model \\
\hline 1 \\
2 \\
3 \\
4
\end{tabular}

\begin{tabular}{c}
\hline Omentin-1 OR $(95 \% \mathrm{Cl})$ \\
\hline $1.34(1.03 ; 1.75)$ \\
$1.41(1.07 ; 1.85)$ \\
$1.42(1.03 ; 1.90)$ \\
$1.40(1.03 ; 1.90)$ \\
\hline
\end{tabular}

\begin{tabular}{c}
\hline $\boldsymbol{P}$ \\
\hline 0.029 \\
0.013 \\
0.020 \\
0.032 \\
\hline
\end{tabular}

\begin{tabular}{c}
\hline Adiponectin OR $(95 \% \mathrm{Cl})$ \\
\hline $0.56(0.43 ; 0.73)$ \\
$0.50(0.36 ; 0.67)$ \\
$0.56(0.41 ; 0.78)$ \\
$0.60(0.42 ; 0.85)$
\end{tabular}

$\begin{array}{r}\multicolumn{1}{c}{\boldsymbol{P}} \\ \hline<0.001 \\ <0.001 \\ <0.001 \\ 0.004 \\ \hline\end{array}$

\begin{tabular}{ll}
\hline $\boldsymbol{P}_{\text {interaction }}$ \\
\hline 0.401 \\
0.328 \\
0.112 \\
0.112 \\
\hline
\end{tabular}

The odds ratios (ORs) refer to a 1-S.D. increase in $\log _{2}$-transformed omentin-1 or adiponectin levels at baseline. $P_{\text {interaction }}$ represents the $P$ value for interaction between omentin-1 and adiponectin in the association with incident type 2 diabetes. Model 1: crude. Model 2: model 1+age, sex, smoking, alcohol consumption, physical activity. Model 3: model 2+BMI. Model 4: model 3+ HDL cholesterol, LDL cholesterol, triglycerides, use of lipid-lowering drugs, hypertension, prevalent myocardial infarction, estimated glomerular filtration rate. 
Third, the associations between omentin-1 and increases in glycaemia were independent of adiponectin, because all effect sizes in this study were mutually adjusted for the other adipokine. This is interesting, because not a single risk factor for type 2 diabetes that was investigated in the KORA F4 study remained significantly associated with omentin-1 after correction for adiponectin (4). Additionally, we found no statistical evidence for an interaction between omentin-1 and adiponectin unlike the EPIC-Potsdam study (5), which will be discussed below.

Fourth, despite a positive correlation between omentin-1 and adiponectin and similarly favourable associations of the two adipokines with diabetes risk factors, both proteins differ in their associations with the development of hyperglycaemia. Results from prospective studies are comparable to results from cross-sectional studies with respect to the direction of associations for adiponectin, whereas the opposite is the case for omentin-1 as discussed above.

Collectively, these results suggest that circulating omentin-1 is an independent risk marker for future increases in glycaemia in older, non-diabetic individuals despite a clear cross-sectional association between higher omentin-1 and a lower risk profile for type 2 diabetes.

\section{Associations between omentin-1, adiponectin and incident type 2 diabetes}

The second main finding of this study is the independent positive association between higher omentin-1 levels and higher risk of type 2 diabetes, while the opposite association was seen for adiponectin. The data are consistent with the observations regarding omentin-1 and the deterioration of glycaemia, whereas for adiponectin it seems that the association with incident type 2 diabetes is less affected by adjustment for confounders than its association with increases in glycaemia. Importantly, the prospective data differ again from the cross-sectional observations in the KORA F4 study, which found no differences in omentin-1 between non-diabetic and diabetic individuals (4). Other studies reported lower omentin-1 levels in people with type 2 diabetes $(3,6,10,12)$, but most of them did not adjust for important covariables such as age, sex, obesity or other cardiometabolic risk factors.

The relationship between omentin-1, adiponectin and incident type 2 diabetes was investigated before in the EPIC-Potsdam study (5). Our data confirm that higher levels of omentin-1 are associated with higher diabetes risk. The EPIC-Potsdam study also reported an interaction between systemic levels of omentin-1 and adiponectin in their association with incident type 2 diabetes. Omentin-1 was related to a higher risk of type 2 diabetes particularly in individuals with high adiponectin levels, whereas no clear risk relation was evident in participants with low adiponectin. However there was no such interaction in the KORA F4/FF4 cohort. The reason for this discrepancy could be related not only to biological differences between both populations, but also to differences in age, sample size and statistical power. In any case, the collective evidence from both studies points towards omentin-1 as risk marker of incident type 2 diabetes, which is independent of established diabetes risk factors.

Since systemic levels of omentin- 1 are also associated with a better profile of cardiovascular risk factors, it is important that two prospective studies have investigated if baseline levels of omentin-1 are linked with the risk of cardiovascular events. Data from the EPIC-Potsdam study showed that higher omentin-1 levels were associated with a higher risk of stroke, but not with myocardial infarction (22). Additionally, higher omentin-1 was related to a higher risk of major cardiovascular events in patients undergoing coronary angiography for the evaluation of coronary artery disease (23). Taken together, these studies suggest that higher omentin-1 levels are associated with higher risk of cardiovascular events despite crosssectional associations of omentin-1 with lower levels of cardiovascular risk factors.

\section{Pathophysiological links between omentin-1 and cardiometabolic risk}

The observation that systemic omentin-1 levels are associated with increases in glucose levels and increased risk of type 2 diabetes raises the question how these data can be reconciled with the often-reported associations of this adipokine with a more favourable cardiometabolic risk profile. Two possible explanations for the findings from the prospective studies are (i) direct effects of omentin-1 that impair glucose homeostasis and increase diabetes risk or (ii) regulatory mechanisms that involve an upregulation of omentin-1 by proinflammatory and/or metabolic triggers that promote the development of type 2 diabetes.

With respect to the first explanation, the fact that we found similar associations between omentin-1 levels and both fasting and 2-h glucose levels, which are differently 
regulated, could point towards detrimental effects of omentin-1 on insulin sensitivity in both the liver and in the skeletal muscle. However we are not aware of published data from experimental studies that would support this hypothesis. In contrast, one study reported that omentin-1 enhanced insulin-stimulated glucose uptake and Akt phosphorylation in human adipocytes (1). The currently available evidence also points towards anti-inflammatory effects of omentin-1 on macrophages and endothelial cells $(24,25,26)$, which would be diabetes-protective rather than diabetogenic, whereas we recently observed an upregulation of proinflammatory cytokines and chemokines by omentin-1 in human adipocytes (27). Moreover, recent reviews summarised several antiatherogenic and cardioprotective effects on endothelial cells, smooth muscle cells and cardiomyocytes ex vivo and in rodent models in vivo (24, $25,26)$, thus arguing against a causal detrimental role of omentin-1 in the establishment and progression of cardiometabolic disease.

Mechanistic in vivo data from humans are currently restricted to small intervention studies using approaches to decrease cardiometabolic risk. Several studies reported that omentin-1 levels in the circulation increased in parallel to lifestyle-induced weight loss (28), after bariatric surgery $(29,30)$ or during exercise interventions $(7,31)$. Thus, these data are more consistent with cross-sectional than prospective associations between omentin- 1 and cardiometabolic risk factors. However it is not clear from these studies if changes in omentin- 1 are causally implicated in lowering cardiometabolic risk or if increases in omentin-1 merely reflect changes in more relevant risk factors such as serum lipids.

The second explanation assumes that the elevated omentin-1 levels at baseline, which indicate a higher risk of type 2 diabetes, reflect an upregulation of omentin-1 by metabolic and/or proinflammatory risk factors that are causally involved in the development of type 2 diabetes. Such a mechanism has been proposed to explain why higher systemic levels of the anti-inflammatory protein interleukin-1 receptor antagonist (IL-1RA) are associated with a higher risk of type 2 diabetes and cardiovascular events despite evidence for diabetic-protective and atheroprotective properties of this protein $(13,32,33)$. For IL-1RA it is assumed that higher levels of IL-1RA reflect the activity of the proinflammatory cytokine IL-1 $\beta$, which is involved in the development of both type 2 diabetes and cardiovascular disease $(13,33,34$, $35)$. In the case of omentin- 1 , data on its regulation are too limited to identify triggers that could be responsible for such an upregulation. The aforementioned reports of cross-sectional associations between higher omentin-1 levels and lower levels of glucose, insulin resistance and cardiovascular risk factors argue against simple reverse causation and illustrate the need for further studies to better understand the regulation of omentin- 1 expression and release.

\section{Strengths and limitations}

Strengths of this study include the prospective design, the availability of OGTT data from both baseline and follow-up examinations to assess changes in glucose tolerance, the rigorous case validation, the use of both changes in quantitative parameters of glycaemia and incident diabetes as outcomes, the direct comparison of omentin-1 and adiponectin, and the adjustment for a large number of potential confounders.

The main limitation of our study is the observational study design that precludes the investigation of mechanisms underlying the regulation of systemic omentin-1 levels. The KORA F4 study is the first follow-up study of the population-based KORA S4 study. Based on the response rate of $70 \%$ in the age group and further dropouts between KORA F4 and KORA FF4, the present study sample is less representative for the initial study population. Since the general state of health of the drop-outs was lower than that of the remaining study participants, we cannot exclude that selection bias affected our results. However participants and non-participants did not differ in their systemic omentin-1 and adiponectin levels. The age range of our study was limited to $62-81$ years so that our results cannot be generalised to younger populations. Additionally, our results may be biased by competing risks if omentin-1 levels were associated with premature mortality, morbidity or other reasons for nonparticipation in the follow-up examination. Changes in methods of measuring glucose and HbA1c between KORA F4 and FF4 were further limitations. Moreover, the ethnic composition of the study population does not allow us to generalise our findings to populations of non-European descent. Finally, despite adjustment for multiple confounders we cannot exclude residual confounding.

\section{Conclusion}

This study observed that higher systemic levels of omentin-1 were associated with increases in fasting glucose, 2-h glucose and HbA1c and with higher risk of 
type 2 diabetes over a follow-up time of 6.5 years in an older population, whereas adiponectin levels showed associations in the opposite direction. The associations for omentin-1 and adiponectin were independent of potential confounders and also independent of one another. Future studies need to elucidate how omentin-1 levels are regulated and the effect of omentin-1 on the body to better understand its role in the development of type 2 diabetes and other diseases.

\section{Supplementary data}

This is linked to the online version of the paper at http://dx.doi.org/10.1530/ EJE-17-0100.

\section{Declaration of interest}

The authors declare that there is no conflict of interest that could be perceived as prejudicing the impartiality of this clinical study.

\section{Funding}

The KORA study was initiated and financed by the Helmholtz Zentrum München - German Research Center for Environmental Health, which is funded by the German Federal Ministry of Education and Research (BMBF) and by the State of Bavaria. Furthermore, KORA research was supported within the Munich Center of Health Sciences (MC-Health), Ludwig-Maximilians-Universität, as part of LMUinnovativ. This work was supported by the Ministry of Science and Research of the State of North Rhine-Westphalia (MIWF NRW) and the German Federal Ministry of Health (BMG). The diabetes part of the KORA F4 study was funded by a grant from the German Research Foundation (DFG; RA 459/3-1). This study was supported in part by a grant from the German Federal Ministry of Education and Research (BMBF) to the German Center for Diabetes Research (DZD) and by a grant from the German Diabetes Foundation (DDS). The funders of the study had no role in study design; data collection; or analysis, interpretation or writing of the report.

\section{Author contribution statement}

C Herder and B Thorand contributed to the study concept and design. C Herder, M Carstensen-Kirberg, C Huth, W Rathmann, A Peters and C Meisinger contributed data. C Herder and J M Kannenberg planned the statistical analysis. J M Kannenberg conducted the statistical analysis. C Herder, C Niersmann, M Carstensen-Kirberg, C Wittenbecher, M Schulze, $\mathrm{M}$ Blüher and $\mathrm{M}$ Roden contributed to the interpretation of the data. C Herder drafted the paper. All authors contributed to, critically revised and approved the final version of the manuscript.

\section{Acknowledgements}

We thank Ulrike Partke (German Diabetes Center, Düsseldorf, Germany) for excellent technical assistance with the omentin-1 and adiponectin measurements.

\section{References}

1 Yang RZ, Lee MJ, Hu H, Pray J, Wu HB, Hansen BC, Shuldiner AR, Fried SK, McLenithan JC \& Gong DW. Identification of omentin as a novel depot-specific adipokine in human adipose tissue: possible role in modulating insulin action. American Journal of Physiology: Endocrinology and Metabolism 2006290 E1253-E1261. (doi:10.1152/ ajpendo.00572.2004)

2 de Souza Batista CM, Yang RZ, Lee MJ, Glynn NM, Yu DZ, Pray J, Ndubuizu K, Patil S, Schwartz A, Kligman M et al. Omentin plasma levels and gene expression are decreased in obesity. Diabetes 200756 1655-1661. (doi:10.2337/db06-1506)

3 Yan P, Liu D, Long M, Ren Y, Pang J \& Li R. Changes of serum omentin levels and relationship between omentin and adiponectin concentrations in type 2 diabetes mellitus. Experimental and Clinical Endocrinology and Diabetes 2011119 257-263. (doi:10.105 5/s-0030-1269912)

4 Herder C, Ouwens DM, Carstensen M, Kowall B, Huth C, Meisinger C, Rathmann W, Roden M \& Thorand B. Adiponectin may mediate the association between omentin, circulating lipids and insulin sensitivity: results from the KORA F4 study. European Journal of Endocrinology 2015172 423-432. (doi:10.1530/EJE-14-0879)

5 Wittenbecher C, Menzel J, Carstensen-Kirberg M, Biemann R, di Giuseppe R, Fritsche A, Isermann B, Herder C, Aleksandrova K, Boeing $\mathrm{H}$ et al. Omentin-1, adiponectin, and the risk of developing type 2 diabetes. Diabetes Care 201639 e79-e80. (doi:10.2337/dc15-2702)

6 Pan HY, Guo L \& Li Q. Changes of serum omentin-1 levels in normal subjects and in patients with impaired glucose regulation and with newly diagnosed and untreated type 2 diabetes. Diabetes Research and Clinical Practice 201088 29-33. (doi:10.1016/j.diabres.2010.01.013)

7 Saremi A, Asghari M \& Ghorbani A. Effects of aerobic training on serum omentin-1 and cardiometabolic risk factors in overweight and obese men. Journal of Sports Sciences 201028 993-998. (doi:10.1080/0 2640414.2010.484070)

8 Catli G, Anik A, Abaci A, Kume T \& Bober E. Low omentin-1 levels are related with clinical and metabolic parameters in obese children. Experimental and Clinical Endocrinology and Diabetes 2013121 595-600. (doi:10.1055/s-0033-1355338)

9 Wang Q, Feng X, Zhou C, Li P \& Kang J. Decreased levels of serum omentin-1 in patients with obstructive sleep apnoea syndrome. Annals of Clinical Biochemistry 201350 230-235. (doi:10.1177/0004563212473275)

10 Greulich S, Chen WJ, Maxhera B, Rijzewijk LJ, van der Meer RW, Jonker JT, Mueller H, de Wiza DH, Floerke RR, Smiris K et al. Cardioprotective properties of omentin- 1 in type 2 diabetes: evidence from clinical and in vitro studies. PLOS ONE 20138 e59697. (doi:10.1371/journal.pone.0059697)

11 Onur I, Oz F, Yildiz S, Oflaz H, Sigirci S, Elitok A, Pilten S, Karaayvaz EB, Cizgici AY, Kaya MG et al. Serum omentin 1 level is associated with coronary artery disease and its severity in postmenopausal women. Angiology 201465 896-900. (doi:10.1177/0003319713511322)

12 Zhang Q, Zhu L, Zheng M, Fan C, Li Y, Zhang D, He Y \& Yang H. Changes of serum omentin-1 levels in normal subjects, type 2 diabetes and type 2 diabetes with overweight and obesity in Chinese adults. Annales d'Endocrinologie 201475 171-175. (doi:10.1016/j. ando.2014.04.013)

13 Herder C, Carstensen M \& Ouwens DM. Anti-inflammatory cytokines and risk of type 2 diabetes. Diabetes, Obesity and Metabolism 201315 (Supplement 3) 39-50. (doi:10.1111/dom.12155)

14 Rathmann W, Haastert B, Icks A, Löwel H, Meisinger C, Holle R \& Giani G. High prevalence of undiagnosed diabetes mellitus in Southern Germany: target populations for efficient screening. The KORA survey 2000. Diabetologia 200346 182-189. (doi:10.1007/ s00125-002-1025-0)

15 Rathmann W, Strassburger K, Heier M, Holle R, Thorand B, Giani G $\&$ Meisinger C. Incidence of Type 2 diabetes in the elderly German population and the effect of clinical and lifestyle risk factors: KORA S4/F4 cohort study. Diabetic Medicine 200926 1212-1219. (doi:10.1111/j.1464-5491.2009.02863.x) 
16 Goek ON, Prehn C, Sekula P, Römisch-Margl W, Döring A, Gieger C, Heier M, Koenig W, Wang-Sattler R, Illig T et al. Metabolites associate with kidney function decline and incident chronic kidney disease in the general population. Nephrology Dialysis Transplantation 201328 2131-2138. (doi:10.1093/ndt/gft217)

17 The Expert Committee on the Diagnosis and Classification of Diabetes Mellitus. Report of the Expert Committee on the diagnosis and classification of diabetes mellitus. Diabetes Care 200326 (Supplement 1) S5-S20. (doi:10.2337/diacare.21.1.s5)

18 Laxy M, Knoll G, Schunk M, Meisinger C, Huth C \& Holle R. Quality of diabetes care in Germany improved from 2000 to 2007 to 2014, but improvements diminished since 2007. Evidence from the population-based KORA studies. PLOS ONE 201611 e0164704. (doi:10.1371/journal.pone.0164704)

19 Herder C, Bongaerts BW, Rathmann W, Heier M, Kowall B, Koenig W, Thorand B, Roden M, Meisinger C \& Ziegler D. Association of subclinical inflammation with polyneuropathy in the older population: KORA F4 study. Diabetes Care 201336 3663-3670. (doi:10.2337/dc13-0382)

20 Herder C, Kannenberg JM, Carstensen-Kirberg M, Huth C, Meisinger C, Koenig W, Peters A, Rathmann W, Roden M \& Thorand B. Serum levels of interleukin-22, cardiometabolic risk factors and incident type 2 diabetes: KORA F4/FF4 study. Cardiovascular Diabetology 20171617. (doi:10.1186/s12933-017-0498-6)

21 Jialal I, Devaraj S, Kaur H, Adams-Huet B \& Bremer AA. Increased chemerin and decreased omentin- 1 in both adipose tissue and plasma in nascent metabolic syndrome. Journal of Clinical Endocrinology and Metabolism 201398 E514-E517. (doi:10.1210/jc.2012-3673)

22 Menzel J, di Giuseppe R, Biemann R, Wittenbecher C, Aleksandrova $\mathrm{K}$, Pischon T, Fritsche A, Schulze MB, Boeing H, Isermann B et al. Omentin-1 and risk of myocardial infarction and stroke: results from the EPIC-Potsdam cohort study. Atherosclerosis $2016251415-421$. (doi:10.1016/j.atherosclerosis.2016.06.003)

23 Saely CH, Leiherer A, Muendlein A, Vonbank A, Rein P, Geiger K, Malin C \& Drexel H. High plasma omentin predicts cardiovascular events independently from the presence and extent of angiographically determined atherosclerosis. Atherosclerosis $2016 \mathbf{2 4 4}$ 38-43. (doi:10.1016/j.atherosclerosis.2015.10.100)

24 Tan YL, Zheng XL \& Tang CK. The protective functions of omentin in cardiovascular diseases. Clinica Chimica Acta 2015448 98-106. (doi:10.1016/j.cca.2015.05.019)

25 Ouchi N, Ohashi K, Shibata R \& Murohara T. Protective roles of adipocytokines and myokines in cardiovascular disease. Circulation Journal 201680 2073-2080. (doi:10.1253/circj.CJ-16-0663)
26 Cheng X. Elucidating the pathophysiological significance of circulating omentin levels: is higher better? Atherosclerosis 2016251 522-524. (doi:10.1016/j.atherosclerosis.2016.06.042)

27 Carstensen-Kirberg M, Roehrig K, Ouwens M, Roden M \& Herder C. Upregulation of proinflammatory chemokines and cytokines in human adipocytes by the adipokine omentin. Diabetes $2006 \mathbf{6 5}$ (Supplement 1) A432.

28 Moreno-Navarrete JM, Catalán V, Ortega F, Gómez-Ambrosi J, Ricart W, Frühbeck G \& Fernández-Real JM. Circulating omentin concentration increases after weight loss. Nutrition and Metabolism 20107 27. (doi:10.1186/1743-7075-7-27)

29 Lapointe M, Poirier P, Martin J, Bastien M, Auclair A \& Cianflone K. Omentin changes following bariatric surgery and predictive links with biomarkers for risk of cardiovascular disease. Cardiovascular Diabetology 201413 124. (doi:10.1186/s12933-014-0124-9)

30 Urbanová M, Dostálová I, Trachta P, Drápalová J, Kaválková P, Haluzíková D, Matoulek M, Lacinová Z, Mráz M, Kasalický M et al. Serum concentrations and subcutaneous adipose tissue mRNA expression of omentin in morbid obesity and type 2 diabetes mellitus: the effect of very-low-calorie diet, physical activity and laparoscopic sleeve gastrectomy. Physiological Research 201463 207-218.

31 Madsen SM, Thorup AC, Bjerre M \& Jeppesen PB. Does 8 weeks of strenuous bicycle exercise improve diabetes-related inflammatory cytokines and free fatty acids in type 2 diabetes patients and individuals at high-risk of metabolic syndrome? Archives in Physiology and Biochemistry 2015121 129-138. (doi:10.3109/13813455.2015.1082600)

32 Carstensen M, Herder C, Kivimäki M, Jokela M, Roden M, Shipley MJ, Witte DR, Brunner EJ \& Tabák AG. Accelerated increase in serum interleukin-1 receptor antagonist starts 6 years before diagnosis of type 2 diabetes: Whitehall II prospective cohort study. Diabetes 2010 59 1222-1227. (doi:10.2337/db09-1199)

33 Herder C, Dalmas E, Böni-Schnetzler M \& Donath MY. The IL-1 pathway in type 2 diabetes and cardiovascular complications. Trends in Endocrinology and Metabolism 201526 551-563. (doi:10.1016/j. tem.2015.08.001)

34 Donath MY. Targeting inflammation in the treatment of type 2 diabetes: time to start. Nature Reviews Drug Discovery 201413 465-476. (doi:10.1038/nrd4275)

35 Herder C, de las Heras Gala T, Carstensen-Kirberg M, Huth C, Zierer A, Wahl S, Sudduth-Klinger J, Kuulasmaa K, Peretz D, Ligthart S et al. Circulating levels of interleukin 1-receptor antagonist and risk of cardiovascular disease: meta-analysis of 6 population-based cohorts. Arteriosclerosis, Thrombosis, and Vascular Biology 201737 1222-1227. (doi:10.1161/ATVBAHA.117.309307)

Received 6 February 2017

Revised version received 16 June 2017

Accepted 4 July 2017 